\title{
Genetic control of diabetes mellitus
}

\author{
Work group chairman: $D$. Weatherall ${ }^{1}$ \\ Report prepared by : N. Sarvetnick ${ }^{2}$ and J. A. Shizuru ${ }^{3}$ \\ ${ }^{1}$ Nuffield Department of Medicine, John Radcliffe Hospital, Headington, Oxford, UK; ${ }^{2}$ Department of Neuropharmacology, Scripps \\ Clinic and Research Foundation, LaJolla, California, USA; ${ }^{3}$ Department of Medicine, Division of Rheumatology and Immunology, \\ Stanford University School of Medicine, Stanford, California, USA
}

Summary. Genetic inheritance predisposing individuals to diabetes mellitus was discussed in this work group. The two forms of the disease, Type 1 (insulin-dependent) and Type 2 (non-insulin-dependent) were discussed separately since the pattern of inheritance and genes involved appear to be distinctly different. Within these subtypes there is considerable genetic heterogeneity, and superimposed environmental factors confound the analysis. New technologies that will allow finer molecular analysis, as well as new candidates genes, were presented.

Key words: Diabetes, genetics, susceptibility.

\section{Introduction}

Diabetes mellitus constitutes a heterogeneous group of disorders characterised by aberration in the production or action of insulin or both. The disease is usually divided into two major subtypes: Type 1 (insulin-dependent) and Type 2 (non-insulin-dependent) diabetes. The aetiology of both forms is thought to be multifactorial-that is, to involve both genetic and environmental components. However, the pathologies of the two appear to be distinctly different. Type 1 diabetes generally occurs in younger persons and is due to the loss of insulin production because of autoimmune destruction directed specifically towards the insulin-producing islet Beta cells. The bulk of evidence indicates that autoreactive $T$ lymphocytes initiate and oversee this Beta-cell specific immune destruction [1]. In contrast, Type 2 diabetes is seen in older and frequently overweight people, and is associated with dysfunction of both insulin secretion and peripheral insulin resistance [2]. Here we summarize the work group on genetic control of diabetes delineating between the two major forms of the disease.

\section{Genetic control of Type 1 diabetes}

Type 1 diabetes is inherited as a complex polygenic trait [3]. Its incidence follows a worldwide north-south geographic gradient with the highest incidence occurring in Scandinavia at approximately 40 per 100,000 children or young adults. Genetic concordance between identical twins is estimated at $30-40 \%$. Environmental factors are thought to trigger the autoimmune destruction of islets in genetically susceptible individuals. The prominent role of the immune system in the pathogenesis of Type 1 diabetes has correlated well with the finding that the one most clearly identified susceptibility gene(s) maps to the class II region of the major histocompatibility complex (MHC). The gene products of the MHC play a pivotal role in antigen recognition and immune system activation.

\section{MHC genes and Type 1 diabetes}

\section{Class II MHC haplotype - an oversimplified view}

To date, the strongest genetic association exists between the class II MHC genes (designated DR in humans and Ia in mice) and susceptibility or resistance to diabetes. Earlier studies demonstrated by serology that certain DR haplotypes were closely associated with Type 1 diabetes. Specifically, in the Caucasian populations the DR3 and DR4 haplotypes were found to have the strongest association with Type 1 diabetes, while DR2 was negatively associated with Type 1 diabetes [4]. However, recent studies and many of the discussions in this work group indicated that this approach is overly simplistic. As pointed out by Erlich, many different DR haplotypes have been noted to contribute to Type 1 diabetes risk; however, significant complexity arises because on a given haplotype (e.g. DR4) there are multiple loci (e.g. DQA1, DQB1, DRB1) that contain multiple alleles (e.g. DRB1.0401, 0402, 
0405). These individual components are capable of contributing both resistance and susceptibility.

\section{MHC population studies}

The work group studies examining different diabetic populations throughout the world underscored the complexities involved in correlating HLA haplotypes with Type 1 diabetes. A recurrent theme in these presentations was that susceptibility alleles defined for one population do not necessarily confer susceptibility in another geographically distinct population. For example, J. Bell presented studies comparing patients and control subjects from the United Kingdom and France in which Type 1 diabetes susceptibility is contributed in the UK group from loci in DR:Dw4, Dw10 and Dw15. However, in the French population these same alleles do not confer susceptibility. Similarly, population studies presented by Serjeantson demonstrated that unlike what has been found for the European (Caucasian) population where HLA-DQB1*0302 is considered a high-risk Type 1 diabetes allele, in Oceania (Polynesia, Australia, etc.) this same allele has not been found to be high risk.

Erlich described work from a study of 41 MexicanAmerican families done collaboratively with Rotter and Zeidler. As with Caucasian Type 1 diabetes, there is an increase in DR3 and DR4. A synergistic effect was noted between DR3 and DR4 since DR3/4 heterozygotes demonstrated the highest relative risk. However, DR3 behaved like a "recessive" allele (two doses were required) so that similar to DR3/4, a DR3/3 genotype conferred risk. In contrast, DR4 behaved like a "dominant" susceptibility gene, since, unlike DR3/X, DR4/X conferred risk.

J. Bell presented additional data from another autoimmune disease, coeliac disease, demonstrating that in certain populations DR3 conferred strong susceptibility, whereas in other populations DR5/DR7 heterozygotes showed strong susceptibility. DNA sequence analysis revealed two determinants on $D Q A$ and $D Q B$, that on the DR3 haplotype are found together in cis, whereas these determinants are present in trans in the DR5/DR7 heterozygotes. He suggested that perhaps DR5 and DR7 might together produce the same heterodimeric susceptibility molecule as is present in DR3. Thus, both cis and trans events occurring within the MHC can potentially contribute to susceptibility (or protection).

\section{Theoretical models of MHC and disease susceptibility}

Thomson presented studies done collaboratively with Klitz in which data analysis and theoretical modelling derived from 811 Caucasian Type 1 diabetic families was used to detect genetic heterogeneity and predict modes of inheritance in Type 1 diabetes. Simplex families or multiplex families were studied. Simplex families consist of unaffected parents with an affected child or children, whereas multiplex families have more than one affected individual, that is, an affected parent with an affected child or children. The rationale for studying simplex families is that they allow the study of internal controls since elements not inherited from normal parents can be examined. The simplex family analysis yielded results that concurred with Erlich's results of Mexican-American families presented above. That is, the DR4/X haplotype behaves like the high susceptibility DR3/4 haplotype, and the DR4 effect appears to act synergistically (acts dominantly) with any other haplotype that is not DR4. Interestingly, however, Thomson notes that this data did not support the notion that DR4 is acting as a simple dominant, since DR4/4 "homozygotes" are associated with lessened susceptibility.

In the multiplex family studies the results were quite different. DR4 was transmitted in excess of Type 1 diabetes frequency such that DR4 appeared less "dominant" in multiplex vs simplex families. Thomson's interpretation of these data is that in multiplex families the parents have a greater number of Type 1 diabetes riskassociated factors, thus the effect of a single susceptibility determinant (i.e. DR4) is lessened relative to other riskassociated loci.

\section{Discussion: MHC genes and Type 1 diabetes}

General discussion of these data involved a number of proposals to explain the observed heterogeneity among different diabetic populations. The consensus among the participants was that fine genetic mapping appears to be a requisite in order to clarify these differences. Susceptibility most likely results from a collaboration of factors which accumulate at the DNA sequence levels. The attempts to consolidate susceptibility haplotypes by using a common marker (e.g. DQA-Arg 52) can give rise to higher relative risk values, but grouping high risk (DR3/4) and lower risk $\mathrm{DR}(3 / 3$ or $4 / 4)$ genotypes gives a lower absolute risk. Thus, rather than examine whole haplotypes, the fine structure (i.e. the nucleotide sequence) of individual alleles must be examined. Erlich pointed out that generalisable information can be obtained by comparing disease susceptibility of two very closely matched alleles that differ by only one residue. The Asp at position DQB-57 is implicated by this analysis, but he cautioned that the DQB57 residue per se should not be used to predict risk. Furthermore, it is possible that susceptibility determinants may be localised to the gene regulatory regions and thus it would be relevant to compare alleles with structurally heterogenous regulatory elements.

\section{Biology of MHC susceptibility}

The way in which the gene products of the associated susceptibility MHC genes influence diabetes pathogenesis is not understood. The function of MHC molecules in normal immune responses is to bind peptide antigen and present this antigen to $\mathrm{T}$ lymphocytes. The response of the $T$ cell(s) may have very different outcomes depending 
upon where and at what stage of T-cell development the encounter with the MHC/antigen complex occurs [5]. It is generally thought that mature circulating $\mathrm{T}$ cells would be activated and elicit other arms of the immune system, whereas immature $T$ cells developing in the thymus would either be destroyed if the antigen constituted a component of self (negative selection), or be allowed to mature if the antigen was foreign (positive selection). Two hypotheses were discussed regarding the role of the MHC gene products in eliciting the autoimmune response.

Nepom supported the notion that Type 1 diabetes susceptibility is correlated with peptide presentation by MHC molecules. He suggested that because a hierarchy for peptide affinity exists among the different MHC molecules, and because MHC molecules are co-dominantly expressed, competition for peptide binding to different MHC molecules might occur within an antigen-presenting cell. Thus, for example, dominant protective MHC molecules might have the highest affinity for a given peptide but be unable to stimulate the pathogenic $T$ cells. This competitive interaction would be dependent upon limiting amounts of antigen in the endosome (unlimited amounts of peptide would result in promiscuous binding). Furthermore, he noted that different MHC alleles appear to be differentially susceptible to up or down regulation. For example, DQ3.2 (DQ8) shows three-fold higher level of expression compared with DQ3.1 (DQ7) by experiments in which the DQ3.2 and DQ3.1 promoters were linked to a reporter gene. It is possible that these regulatory effects may be tissue specific in vivo. Thus, from this example it appears that changes in recognition sequences for transcription factors can occur in the different haplotypes. These effects might cause an enhancement of antigen presentation in specific tissues.

J. Bell discussed studies involving linkage between certain T-cell receptors (TCR) and susceptibility to Type 1 diabetes. Others have shown no linkage between Type 1 diabetes with TCR a or b chains. However, J. Bell has examined the allelic variation in TCR genes utilising PCR amplification and electrophoresis of denatured DNA sequences. He has derived large fragments of the genome from TCR variable regions and has been isolating repetitive sequences which have been utilised to study disease associations. Thus far he has found no significant association between TCR and disease. These techniques of using denaturing gels allow an analysis of single residue polymorphisms rather than clustered polymorphisms.

\section{Non-MHC genes in human diabetes}

Todd presented an update on progress in screening the human genome for susceptibility genes outside the MHC and insulin genes. This work complements the studies carried out in the mouse system described below. The approach has been to screen for regions by (a) using randomly selected, highly polymorphic microsatellites and (b) restriction length fragment polymorphisms (RLFPs) and microsatellites in or near candidate genes by linkage analysis and association studies. For these strategies, the following have been collected (a) 140 multiplex families with at least two affected siblings (proband age-onset less than 17 years) and both parents available, (b) 300 sibling (isolated cases) Type 1 diabetic patients and (c) 250 unrelated healthy control subjects. All these individuals are ethnically matched with grandparents born in the UK. A collection of more than 110 multiplex families is also available from the Human Biological Disease Interchange (HBDI) in Philadelphia, Pennsylvania, USA.

\section{Rodent models of Type 1 diabetes}

Genetic mapping of the human diabetes susceptibility genes is currently being carried out in parallel with rodent models of spontaneous autoimmune diabetes: the nonobese diabetic mouse (NOD) and the biobreeding (BB) rat. Disease pathology in these rodents has many features in common with human Type 1 diabetes, including autoimmune islet destruction possibly directed by $T$ lymphocytes and associated with islet antibodies, as well as the presence of susceptibility genes in the MHC $[1,3]$. As with humans, there is a lack of complete genetic concordance despite the fact that rodents are inbred, and the environment impacts upon the development of diabetes. $B B$ rats and NOD mice kept in a specific pathogen-free environment were reported to have an increased frequency of diabetes.

\section{The murine MHC and diabetes susceptibility}

In agreement with the findings in human Type 1 diabetes, it has been shown that NOD mice express a single unique class II molecule designated I-A $\mathrm{g}^{\mathrm{7}}$. Amino acid sequence analysis has revealed that, similar to the human class II molecule, the NOD I- $\mathrm{A}^{\mathrm{g}^{7}}$ molecule differs from other strains of mice in the amino acids in or around residue 57 [5]. As is the case in susceptible human alleles, the NOD class II molecule has a serine and not an aspartate at that residue. In order to test the effect that introduction of a modified or allogeneic class II molecule might have on NOD diabetes, several transgenic mice were generated that expressed unmanipulated $a$ and $b$ transgenes [6]. These transgenic mouse studies demonstrated that non-NOD I-A ${ }^{k}$ transgene expression dramatically reduced insulitis and prevented diabetes. In addition, transgenic NOD mice made to express a second murine class II molecule, the I-E molecule (which NOD mice lack), confirmed a previous report that expression of I-E (also a transgene) can protect NOD mice from development of disease.

Wicker presented a series of very interesting studies using a classic genetic approach in which the NOD MHC class II region was replaced either completely or in part by the $\mathrm{MHC}$ regions of other mouse strains. In the first experiments, the entire NOD MHC region was replaced. 
Table 1. Incidence of thyroiditis in an NOD MHC congenic strain

\begin{tabular}{lccc} 
& NOD & B10. A(4R) & NOD. H-2 \\
\hline $\begin{array}{l}\text { \% thyroiditis } \\
\text { (spontaneous) }\end{array}$ & $5 \%$ & $0 \%$ & $50 \%$ \\
K1 added to diet & $5 \%$ & $0 \%$ & $90 \%$ \\
\hline
\end{tabular}

NOD mice made congenic for the MHC of C57BL/10 on the NOD background (NOD-H.2 $2^{\text {b }}$ ) were generated to test the effect of MHC on diabetes and insulitis. None of NOD$\mathrm{H} .2^{\text {b }}$ became diabetic; however, a non-productive (perivascular and periductal) islet inflammation was noted in several congenic mice by 6 months of age. Immunohistochemical analysis of the cell type in the lesions revealed that the early lesions contained a predominance of CD4+ and CD8+ cells; however, as the mice aged, the infiltrate was largely replaced by $B$ lymphocytes. In addition, interleukin-2 receptor expression also decreased with time. These mice were also noted to have salivary gland inflammation (sialitis), antipolar antigen antibodies and antibodies to salivary glands. Wicker suggested that these observed immune-associated abnormalities support the concept of "autoimmunity" genes outside the MHC.

In crosses of these NOD-H. $2^{b}$ congenics with NOD to generate (NOD x NOD-H. $2^{b}$ )F1 mice, Wicker noted that $2 \%$ of the mice developed spontaneous Type 1 diabetes, $40 \%$ developed insulitis, and administration of cytoxan resulted in overt diabetes in $25 \%$ of the mice. Cytoxan is an alkylating drug that has been shown to induce or accelerate diabetes in subdiabetic or young NOD mice that already have insulitis. These data suggest that the MHC of NOD acts dominantly and that penetrance varies according to the phenotype examined.

Wicker then presented data on other MHC congenic NOD lines expressing I- $E^{k}$ and/or I-A $A^{k}$. F1 crosses of NOD.B10.BR (which will express I-Ek and I-A $\mathrm{A}^{\mathbf{k}}$ ) $\mathrm{NOD}$ demonstrated insulitis, cytoxan-induced diabetes and spontaneous diabetes in some that were 10 months of age or more. These studies are in contrast to the transgenic studies described above where expression of an I-E molecule conferred disease protection. These data suggest that when endogenous I-E molecules are expressed in NOD-congenic mice, their effect is not as protective as seen when the I-E molecule is expressed as transgene.

The third NOD MHC congenic line presented by Wicker expresses I-A $A^{k}$ but is I-E negative. These mice were generated by crosses of NOD with B10A.4R (NOD.H-2 ${ }^{k 4}$ ) and only approximately $20 \%$ of the mice develop insulitis, and (as expected since no dose of MHC of NOD is present) none develop disease. Interestingly, these congenic mice develop thyroiditis at a high rate (and greater than NOD mice).

Wicker suggested that the NOD non-MHC genes may function as "autoimmunity" genes, while the MHC influences or directs which autoimmunity syndrome will predominate. In this example $1-A^{k}$ is the permissive allele for experimental thyroiditis. Wicker cited a recent study indicating that an iodinated peptide from thyroglobulin is a pathogenic epitope in the context of I-A $\mathrm{A}^{\mathrm{k}}$.

\section{Murine non-MHC genes}

Recently, Todd, Wicker and colleagues used locus specific microsatellites to screen the mouse genome for diabetes susceptibility genes in (NOD $\times$ C57BL/10.H- $2^{87}$ ) F1 $\times$ NOD mice [7]. They reported here that at least seven different loci outside the MHC (on chromosome 17) on chromosomes $1,3,4,5,6,11$ and 14 were diabetes associated. Wicker stressed that while there are many genetic effects determining diabetes, there are some genes contributed from the $\mathrm{B} 6$ and $\mathrm{B} 10$ backgrounds that protect from diabetes when transferred to the NOD.

Lathrop presented results of genome screening from another second backcross of NOD mus spretus (a different mouse subspecies) demonstrating that there are at least six non-MHC linked loci that confer susceptibility. Provocatively, these genes do not correlate with the genes mapped in the above NOD x B10 crosses.

\section{Mathematical modelling of diabetes susceptibility genes}

Risch used the NOD $x$ C57BL/10 backcross data to test the mode of Type 1 diabetes inheritance as applied to mathematical models.

In an additive model there are no interactive effects between the various genetic loci, whereas in a multiplicative model there are interactive effects (epistases) between the loci. The mouse backcross data suggested that many loci are involved in susceptibility to NOD between the two strains (at least 10 loci identified so far). These loci show strong interactive effects (epistases) increasing the risk to NOD between NOD homozygotes versus heterozygotes. Both a multiplicative-type epistasis model and a polygenic threshold type model seem to give an adequate explanation of the data, although both models appear to underestimate the degree of epistasis among loci.

In translating from NOD to human Type 1 diabetes, Risch cautioned that one cannot predict how important any of these loci will be in human Type 1 diabetes (in terms of familial aggregation and linkage) because genetic impact depends more on the allelic frequencies (impossible to predict) than observed risk ratios for homozygotes/heterozygotes.

\section{Discussion: genetic control of murine diabetes}

A consensus opinion of the work group was that multiple loci confer susceptibility or resistance or both to diabetes and these will become penetrant in the different strain combinations. Therefore, backcross experiments from individual strain combinations cannot be used to draw universal conclusions, since some of these appear to be non-overlapping genes. Based upon the non-commonality of susceptibility genes mapped in the two strains described above, non-MHC genes appear to involve a constellation of 
different genes; thus it would be important to continue to pursue the biology of islet destruction parallel with the development of further strain combinations aimed more clearly at mapping additional susceptibility genes.

\section{Type 2 diabetes}

Type 2 diabetes affects about $6 \%$ of the population by age 50 and $11 \%$ of the population by age 70 . Evidence for a strong genetic component includes the high concordance rate among identical twins (most studies indicate more than $90 \%$ concordance, although one indicates only $55-60 \%$ concordance), and strong family clustering of disease. Type 2 diabetes occurs by age 60 in $20 \%$ of offspring of one diabetic parent, over $50 \%$ of offspring in two Type 2 diabetic parents, and between $20 \%$ and $40 \%$ of siblings of diabetic probands [2]. Despite this, our understanding of the genetics of Type 2 diabetes is very limited. A number of factors confound the analysis of simple family studies. These include the late onset of disease, lack of well-defined phenotypes, and the difficulty of collecting multigeneration pedigrees, since often the parents and other previous generation relatives may be deceased by the time of identification of the probands.

One form of Type 2 diabetes which is more amenable to study is the so-called "maturity-onset diabetes of youth" or MODY [2]. MODY is generally defined as Type 2 diabetes occurring in three consecutive generations with early age of onset, usually under 45 years of age, although many pedigrees contain affected individuals with onset at under 25 years of age. There remains a debate as to whether MODY is a unique autosomal dominant form of the disease, or simply one end of the spectrum of Type 2 diabetes. Three workshop participants presented results of studies in Type 2 or MODY type diabetes.

Lathrop reported the results of studies from 16 families with MODY diabetes in France. Three candidate genes were investigated: GLUT 2, glucokinase (GCK) and adenosine deaminase (ADA). GLUT 2 and glucokinase, respectively, are uniquely expressed in Beta cells and liver, and are thought to play an important role in Beta-cell sensing of glucose. Although ADA is not thought to directly play a role in the pathogenesis of Type 2 diabetes, it was chosen as a candidate gene based on a previous observation by $\mathrm{G}$. I. Bell that ADA was linked to diabetes in one MODY family [8]. In the French families a most striking linkage was noted between GCK on chromosome $7 p$ [9]. Linkage was observed in $75 \%$ of the families studied, with strong linkage (LOD score $>3$ ) in three families. A linkage to ADA (chromosome 20) was identified in two other families, but there was no linkage to GLUT 2 in seven families studied.

During discussion of this work, G. I. Bell noted that he has identified actual sequence changes in GCK in patients from four MODY kindreds, suggesting that the linkage is due to mutations in the GCK gene. It was also noted that, in the French pedigrees, diabetes is defined using a much "looser" criteria than recommended by WHO or the National Institutes of Health (Bethesda, Md., USA).

Additional studies of MODY families in the UK were reviewed by Wainscoat. Using repeated DNA sequences as markers, one family out of two studied revealed an association of GCK and diabetes. No disease association was found with GLUT or islet-associated pancreatic polypeptide (IAPP) in two and four families, respectively. Wainscoat also reported a rapid strategy for screening for mutations in the insulin promoter region and presented preliminary data for one patient with abnormalities in this region. He also reviewed the technical difficulties of extending these studies to more random Type 2 diabetic patients due to the difficulty in obtaining samples in multigeneration families.

G. I. Bell reviewed his many studies in searching for diabetes-related genes in Type 2 diabetes and MODY families, listing over 15 potential candidate genes for which cDNA clones already exist. In one very large MODY family, significant linkage between the ADA locus and diabetes was noted. It is clear, however, that ADA is only a marker and the actual gene is located telomeric to the ADA locus. Several probes in this area, as well as YAC clones, are being used to localise this gene by positioned cloning.

During the discussion, Kahn pointed out that the pathophysiology of Type 2 diabetes has two components: insulin resistance and the failure of the Beta cells to compensate for the resistance [10]. Thus far, none of the above studies have directly assessed insulin sensitivity in the MODY families and only one has attempted to correlate the findings with changes in insulin secretion. The ADA linkage in the Michigan MODY family correlates with low insulin secretion. Insulin levels in the MODY families associated with GCK have not yet been assessed. Future studies, therefore, should correlate the presumed gene defects with specific physiological abnormalities. It was also pointed out that in pre-Type 2 individuals, insulin resistance may be demonstrated 15 years prior to the onset of clinical disease, while defects in glucose-stimulated insulin release do not appear until a time much closer to the onset of clinical disease.

\section{Conclusions and future directions}

The pathologies of the two forms of diabetes are distinctly different. However, both diseases involve a complex interplay between multiple genetic components and environmental factors. At present the analysis of Type 1 diabetes appears more straightforward since, unlike Type 2 diabetes, the aetiology is understood to be autoimmune. Efforts can therefore be directed towards analysis of genes that control or permit immune destruction of pancreatic islets. Still, as was amply discussed in this workshop, the biology of the autoimmune response is very complex since 
it involves an array of interactive gene products (some contributing to susceptibility and others to resistance), and even very small changes at the DNA sequence level can alter the contribution of a particular gene to Type 1 diabetes pathology. A summary of future directions for genetic analysis of Type 1 diabetes discussed in this workshop are as follows:

- The strongest genetic association exists between the class II MHC genes and susceptibility or resistance to diabetes. However, substantial heterogeneity exists between different human diabetic populations. Since susceptibility most likely results from a collaboration of factors which accumulate at the DNA sequence level, fine genetic mapping appears necessary. More generalisable information might be obtained by comparing disease susceptibility between two very closely matched alleles that differ by only one residue.

- It is possible that susceptibility determinants may be localised to the gene regulatory regions. This point was brought out in discussions of the biology of MHC susceptibility during which it was suggested that differing levels of gene expression among different haplotypes and in different tissues might affect antigen presentation. Thus, it would be relevant to compare alleles with structurally heterogenous regulatory elements.

- Screening of the human and mouse genome for susceptibility genes lying outside the MHC and insulin genes will continue using randomly selected, highly polymorphic microsatellites, and microsatellites in or near candidate genes by linkage and association studies. We can expect to be updated on the progress of these studies in the future.

- Development and implementation of technologies were discussed that allow more sensitive techniques to identify allelic variations, i.e. polymerase chain reaction amplification and electrophoresis of denatured DNA sequences. Given that autoantigen presentation and recognition are dependent upon the fine specificity of immune "receptor" molecules, we can expect further developments in technologies that allow delineation at the DNA sequence level.

- The development of polycongenic mice which contain the susceptibility regions from the NOD on the background of a non-susceptible strain will continue. However, crosses between diabetes-susceptible NOD mice and nonsusceptible strains have indicated that the many loci contributing to susceptibility or resistance or both are variably penetrant depending upon the strain combinations used. Therefore it is important that the biology of islet destruction is studied parallel with the development of further strain combinations aimed at mapping the remaining susceptibility genes.

- Although Type 2 diabetes appears to have a stronger genetic concordance than Type 1 diabetes, genetic analysis of Type 2 diabetes in humans is confounded by the very slow evolution of the disease (generally occurring in older people) and thus difficulty in collecting multi-generation pedigrees, and the variability of phenotype. Furthermore, unlike Type 1 diabetes, the pathophysiology of Type 2 diabetes involves two components - insulin resistance and the inability of the Beta cell to compensate for the insulin resistance - and the molecular events that trigger these changes are not known. The study of the MODY form of the disease has been more amenable to genetic analysis and thus far most of the studies have examined MODY pedigrees. Plans and suggestions for the future include studies that would correlate presumed gene defects with specific physiological abnormalities. For example, thus far none of the MODY families have been assessed for insulin sensitivity although at least three candidate genes have been investigated. The search for diabetes-related genes in Type 2 diabetes and MODY families will continue utilising new technologies as they become available.

Acknowledgements. This report is the result of the Work Group 1: Genetic Control at the Third JDF World Conference on Diabetes in Monte Carlo, Monaco, 8-10 March, 1992. Chairman: D. Weatherall. Work group participants: G. I. Bell ${ }^{1}$, J. Bell ${ }^{2}$, H. A. Erlich ${ }^{3}$, M. Lathrop ${ }^{4}$, G. T. Nepom $^{5}$, N. Risch ${ }^{6}$, S. W. Serjeantson ${ }^{7}$, G. Thomson ${ }^{8}$, J. A. Todd ${ }^{9}$, L. S. Wicker $^{10}$, J. Wainscoat ${ }^{11}$. Rapporteurs: N. Sarvetnick, J. A. Shizuru. Discussants: L. Ducat ${ }^{12}$, B. Singh ${ }^{13}$.

${ }^{1}$ Howard Hughes Medical Institute, University of Chicago, Chicago, Illinois, USA; 'Institute of Molecular Medicine, John Radcliffe Hospital, Headington, Oxford, UK; ${ }^{3}$ Cetus Corporation, Emeryville, California, USA; ${ }^{4} \mathrm{CEPH}$, Paris, France; ${ }^{5}$ Virginia Mason Research Center, Seattle, Washington, USA; ${ }^{\circ}$ Department of Epidemiology and Public Health, Yale School of Medicine, New Haven, Connecticut, USA; ${ }^{7}$ Human Genetics Group, John Curtin School of Medical Research, Canberra, Australia; ${ }^{8}$ Department of Integrative Biology, University of Califomia, Berkeley, Califormia, USA; ${ }^{9}$ Nuffield Department of Surgery, John Radcliffe Hospital, Headington, Oxford, UK; ${ }^{10}$ Merck Sharp \& Dohme Research Laboratories, Rahway, New Jersey, USA; ${ }^{11}$ Haematology Department, John Radcliffe Hospital, Headington, Oxford, UK; ${ }^{12}$ National Disease Research Interchange, Chicago, Illinois, USA; ${ }^{13}$ Department of Immunology, University of Alberta, Edmonton, Alberta, Canada.

\section{References}

1. Eisenbarth GS (1986) Type I diabetes mellitus. A chronic autoimmune disease. N Engl J Med 314:1360-1368

2. Van d'Heim C, Rimoin DL, Rotter J (1990) In: Emery AEH, Rimoin DL (eds) Principles and practice of medical genetics II. Churchill Livingstone, New York

3. Krolewski AS, Warram JH, Rand LI, Kahn CR (1987) Epidemiologic approach to the aetiology of type I diabetes mellitus and its complications. N Engl J Med 317:1390-1392

4. Erlich HA (1990) HLA class II polymorphism and genetic susceptibility to insulin-dependent diabetes mellitus. Curr Top Microbiol Immunol 164:41-43

5. Acha-Orbea H, McDevitt HO (1990) The role of class II molecules in development of insulin-dependent diabetes mellitus in mice, rats and humans. Curr Top Microbiol Immunol 156:103-105

6. Shizuru JA, Sarvetnick N (1990) Transgenic mice for the study of diabetes mellitus. Trends Endocrin Metab 2:97-105

7. Todd JA, Aitman TJ, Cornall RJ et al. (1991) Genetic analysis of autoimmune type I diabetes mellitus in mice. Nature 351:542-546

8. Bell GI, Xiang K-S, Newman MV et al. (1991) Gene for non-insulin-dependent diabetes mellitus (maturity-onset diabetes of the 
young subtype) is linked to DNA polymorphism on human chromosome 20q. Proc Natl Acad Sci USA 88:1484-1488

9. Froguel $\mathrm{Ph}$, Vaxillaire $\mathrm{M}$, Sun $\mathrm{F}$ at al. (1992) Close linkage of glucokinase locus on chromosome $7 p$ to early-onset non-insulindependent diabetes mellitus. Nature 356:162-164
10. Wartam JH, Martin BC, Krolewski AS, Soeldner JS, Kahn CR (1990) Slow glucose removal rate and hyperinsulinemia precede the development of type II diabetes in the offspring of diabetic parents. Ann Intern Med 113:909-921 